\title{
¿Cómo la gente previene enfermarse? Un acercamiento para comprender la forma en que la gente de una comunidad mapuche protege su salud ${ }^{(l)}$
}

Noelia G. Carrasco H. Antropóloga.

\section{RESUMEN}

En este artículo se presenta la síntesis de una investigación antropológica de carácter etnográfica, llevada a cabo entre 1997 y 1998 en una comunidad indígena de la LX Región. Proyectando intereses desde la antropología, la búsqueda por conocer el nivel en que se encuentra la prevención de las enfermedades y la gente vive el cuidado de su salud, este trabajo, que tuvo la forma de investigación de Tesis de Grado, se llevó a cabo a través de la inserción de quien escribe en la comunidad, recogiendo desde allí todo lo que antropológicamente hablara acerca del problema planteado.

El contacto con la gente a través de un acceso cuidadoso a sus distintos contextos de vida, se vio reforzado con el aporte de una persona de la comunidad, que asumió ser mi compañía durante las visitas y conversaciones que constituyeron el nicleo de este trabajo. Esta posibilidad de conformación de un equipo intercultural, se plasmo también en los resultados finales de la etnografia, en donde se presenta una combinación entre las concepciones propias de la gente recogidas desde ellos, y las interpretaciones basadas en un concepto de cultura flexible y dinámico en que se distingue el ser y actuar de la gente, en el ordenamiento y la conjunción de sus ideas y creencias.
Así, la prevención resulta constituir un tipo de comportamiento que existe en todas las sociedades $y$ culturas, y que presenta por tanto, diferentes formas $y$ contenidos. En el caso indagado, pudimos encontrarnos con que la prevención se encuentra ligada íntimamente a otras concepciones, que simultáneamente vienen también a dar cuenta de la prevalencia de un concepto de cultura indígena vigente, en donde los mundos que componen la cosmovisión propia tienen permanencia, entrelazando lo natural con lo sobrenatural, teniendo incidencia directa en la vida $y$ cotidianeidad de la gente. El enfoque de este trabajo situó a la antropología cultural frente a un concepto de ecosistema, asumiendo a este último con contenidos integrados entre las dimensiones medio ambientales, sociales y culturales de la vida.

La comunidad en que se realizó la etnografía de la cual habla este artículo, se encuentra ubicada en el sector de Maquewe, al Sur Oeste de la ciudad de Temuco, Chile. Está conformada por 16 grupos, pertenecientes a 7 troncos familiares, también definidos por la antropología social como familias extensas.

La Tesis realizada intentó reconocer, describir e interpretar las dimensiones sociales y culturales de la vida en la comunidad. Para ello, la metodología etnográfica basada en presupuestos epistemológicos ideográficos, se refundó en la inquietud por hacer una antropología capaz de estar con la gente y comprender su estilo de vida propio, proyectando luego un 
camino de acercamiento y acción antropológica aplicada con comunidades del tipo descrito.

A grandes rasgos, esta investigación concluyó en lo social, con la idea de que esta comunidad sería representativa de un estilo de vida mapuche actual, caracterizada por:

«No poseer una estructura social y política de acuerdo a las formas tradicionales de ordenamiento y poder en torno a la figura político - religiosa del longko, ni tampoco haber consolidado una organización de carácter funcional en su interior, de tal forma que el orden social estaría supeditado a los niveles familiares... La dimensión cultural le estaría otorgando entonces, la cohesión y el ordenamiento que en tanto comunidad, personas y familias mapuches poseen... Este estilo de. comunidad indígena, se caracteriza además por su permanencia en el tiempo y la continuidad de su cohesión a través de la vivencia de sus relaciones internas y externas, asumidas en forma particular por las familias... el control social se expresa a través de las expresiones de voluntad frente a estímulos proporcionados por la sociedad nacional.»(2)

$\mathrm{La}$ indisolubilidad entre las dimensiones sociales y culturales de la vida, dieron origen en esta investigación a la secuencia de argumentación entre un aspecto y otro. La prevalencia de la cultura superaba el desprendimiento de patrones sociales y políticos explícitamente mapuches. Vale decir, pese al desvanecimiento del orden social de la comunidad en torno a la autoridad política y religiosa del longko - durante la década de 1940 - en ella se conserva vigente el entramado cultural mapuche, revelado en esta oportunidad a partir de las concepciones de salud y los comportamientos preventivos de la gente, también comprendido como el circuito conceptual y empírico de medioambiente hombre - salud.

La interrelación entre la gente y su medio ambiente o écosistema, implica consideraciones, creencias y comunicación entre ambos. Habiendo identificado como componentes relevantes de este ecosistema al agua en sus diferentes formas, los bosques originarios, y la tierra en su condición de plataforma integral de la cultura que por tanto, incluye el hombre, el acercamiento etnográfico pudo constatar que en la interacción de la gente con este ecosistema, se encuentran los espacios preventivos, visualizados en el nivel del comportamiento de la gente desprendido de sus concepciones indígenas aun vigentes.

La invéstigación describió a los componentes antes enunciados de la siguiente forma:

«La concepción indígena de mapu, fue la que consensualmente la gente de la comunidad describe como la tierra y sus componentes; lo que de ella se desprende y en ella vive. Allí podemos encontrar el agua, los árboles, las siembras. La gente llamó a todo esto mapu, y a su salud o buena condición tremon mapu»(3)

«Bajo la denominación «ko», el agua representa básicamente la vida, la energía estimulada por el movimiento. Su existencia no puede desprenderse de la existencia de la vida natural (vegetal, animal y humana) y también sobrenatural... siendo un elemento dueño de energía que vitaliza, posee también la doble connotación axiológicamente vivida en la cultura mapuche, aquella que descubre y establece la acción de diversas fuerzas a través del agua... la gente reconoce y comenta acerca de las diferentes fuentes y formas de agua que existen, no se trata de un conocimiento simple, sino por el contrario, profundo y directamente derivado de esta intimidad entre los hombres y el mapu»(4).

«Quedan aun en determinados sectores de la comunidad, y en las propiedades de solo algunos, restos de densos bosques originarios, denominados por la gente como pitrantu, haciendo alusión al arbusto pitra, estos bosques cuentan además con árboles de temu y canelo. Son bosques de tierras húmedas, rodeados de mallines....)(5)

El que cada uno de los componentes antes mencionados posean su vida propia y que esta vitalidad sea

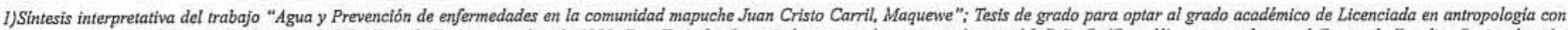

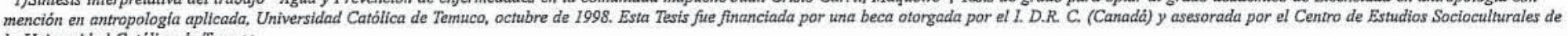
la Universidad Católica de Temuco.

2) Cita extraida del texto de Tesis antes mencionado.

3) Ibid.

4)Ibid.

5)Ibid.
} 
también canalizada a través de la interacción entre lo natural y lo sobrenatural, deja entrever la transversalidad de la vida entre ellos.

La investigación desarrollada se dirigió a indagar acerca de la salud, descubriendo que cada uno de estos elementos poseen su propia salud y que el bienestar de uno estimula el bienestar del otro, o por el contrario, la enfermedad de uno puede también derivar en la enfermedad de otro. El hombre y el ecosistema se encuentran indiscutiblemente insertos y entrelazados en esta red dinámica de salud y bięnestar.

Desde este punto de vista, se pudo también constatar a través de esta etnografía, que el modelo de salud mapuche opera en la comunidad a través de la experiencia y el comportamiento de la gente. Así también, los tipos de enfermedades que la gente sufre, permitieron distinguir que el modelo occidental de salud - enfermedad igualmente opera en determinados casos de enfermedades que la gente denomina «wingka kutran» o enfermedades no mapuches. Este tipo de coexistencia conceptual y también operativa, es una característica ya descrita por otros autores como parte de una situación más o menos generalizada en la sociedad y cultura mapuche actual (6).

De tal forma, se ha podido interpretar que dentro de las concepciones generales de salud, la gente maneja $y$ vive diferentes tipos de enfermedades que se entrelazan a su vez con distintas formas de prevención y cuidado, que provienen de distintos modelos de pensamiento y de salud. Independiente de esta diferenciación, la búsqueda de una buena salud es generalizada...

«... es crucial para la propia subsistencia; poder recoger leña para el fuego y cocinar, ir en busca de agua, cuidar los animales y trabajar la tierra son las actividades cotidianas y culturalmente primordiales. El estado de enfermedad deriva en la imposibilidad de cumplir con estas actividades, valerse por sí mismo y finalmente mantener la vida....)(7).

Retomando las particularidades del modelo mapuche de salud que se vive en la comunidad, las enfermedades mapuches 0 «mapuche kutran» vividas directa e indirectamente por la gente, permitieron elabo- rar una interpretación que vincula al hombre y sus acciones con la dimensión normativa del comportamiento ideal. Esta interpretación se sustenta en la reiteración que muestran los relatos acerca de lo que los afectados debieron y lo que no debieron hacer para haber evitado contraer tal enfermedad. Luego, desde el punto de vista antropológico es posible sostener que...

«El conocimiento cultural en torno a cómo poder prevenir enfermarse o que a alguna persona le afecte un daño causado por otra, apela a la vivencia de situaciones de riesgo que sólo se dejan de correr, cuando la persona y sus más próximos viven en la tranquilidad de que no se han cometido acciones que puedan incitar a otros a hacerles daño... la gente posee un marco de referencia propio para vivir sus relaciones con otra gente y con su ecosistema, entendiendo que este también posee vida y por tanto, al igual que los seres humanos, también es fuente de intencionalidad»(8).

Las situaciones de riesgo son fruto de los comportamientos cometidos. Las atribuciones de culpa y responsabilidad de las enfermedades recaen en las personas una vez que examinan sus acciones y experiencias previas, en donde pudo haber actuado de manera tal que ha provocado la reacción de otros seres naturales o sobrenaturales - sobre ellos mismos. El riesgo o el peligro de sufrir alguna enfermedad está supeditando a los comportamientos, en tanto estos se manifiestan o no acordes con el modelo ideal, normativamente pautado dentro de la cultura. Luego, se expresa también un tipo de dimensión sociomoral, en donđe las acciones son catalogadas y enjuiciadas por el resto de los miembros de la comunidad, quienes con esta misma mirada normativa, valoran positiva o negativamente el comportamiento de los otros, reconociendo en qué momento y con qué actitud se podría haber evitado la enfermedad.

Expresiones de la gente tales como «no debimos haber vivido aquí, tendríamos que haber vivido en la comunidad de mi esposo», o bien «no debí haber tocado a ese animal», denotan que la lógica del riesgo y el peligro de contraer enfermedades se manifiesta en el entramado sociocultural de la comunidad. 
En este sentido, la Tesis pudo explicitar que la prevención posee un nivel sociocultural en tanto se expresa y vivencia a través de los temores de la gente. Este componente del temor, resulta ser fundamental en cuanto al reconocimiento de las situaciones de riesgo culturalmente asumidas por esta. El respeto por no alterar los espacios de vida de otros seres que habitan en lugares como los pitrantu, indica que existe temor por que luego las consecuencias de una alteración premeditada o no, recaigan sobre quien las ejecutó. El miedo o temor de no cometer actos que puedan desencadenar efectos negativos - directos o indirectos- sobre el individuo o sus más cercanos, constituye una dimensión del concepto de salud antes enunciado, que igualmente se puede entender como una elemento regulador de las relaciones e interrelaciones entre los sujetos y su ecosistema.

Según lo dicho por la antropóloga Mary Douglas, las transgresiones explican las desgracias: «... en todos los lugares y épocas el universo se ha interpretado en términos morales....)(9). Serían entonces, lo que ella denomina como «mecanismos de atribución de la culpa» los que varían de una cultura a otra, adheriéndose en cada caso a las particularidades cosmovisionales y de interacción que posean.

En el caso de la comunidad mapuche en que se desarrolló esta etnografía, de acuerdo al enfoque antropológico simbólico - interpretativo, fue posible recoger la vivencia de un modelo de salud vinculado al ecosistema en términos de indisolubilidad e interacción:

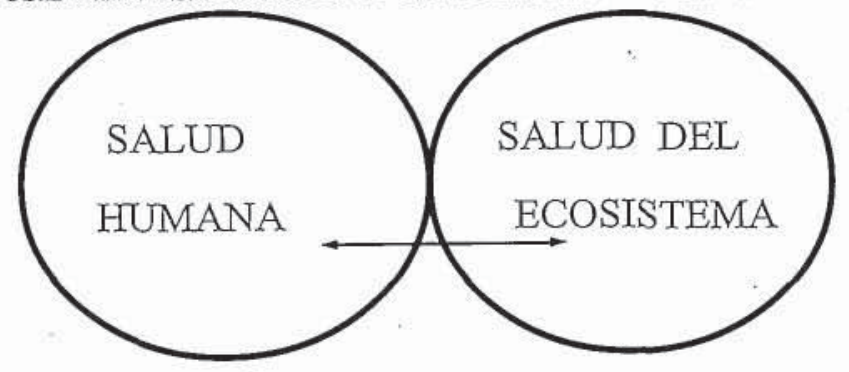

Indisolubilidad en términos de modelo cultural de salud que vincula a lo físico con to espiritual;

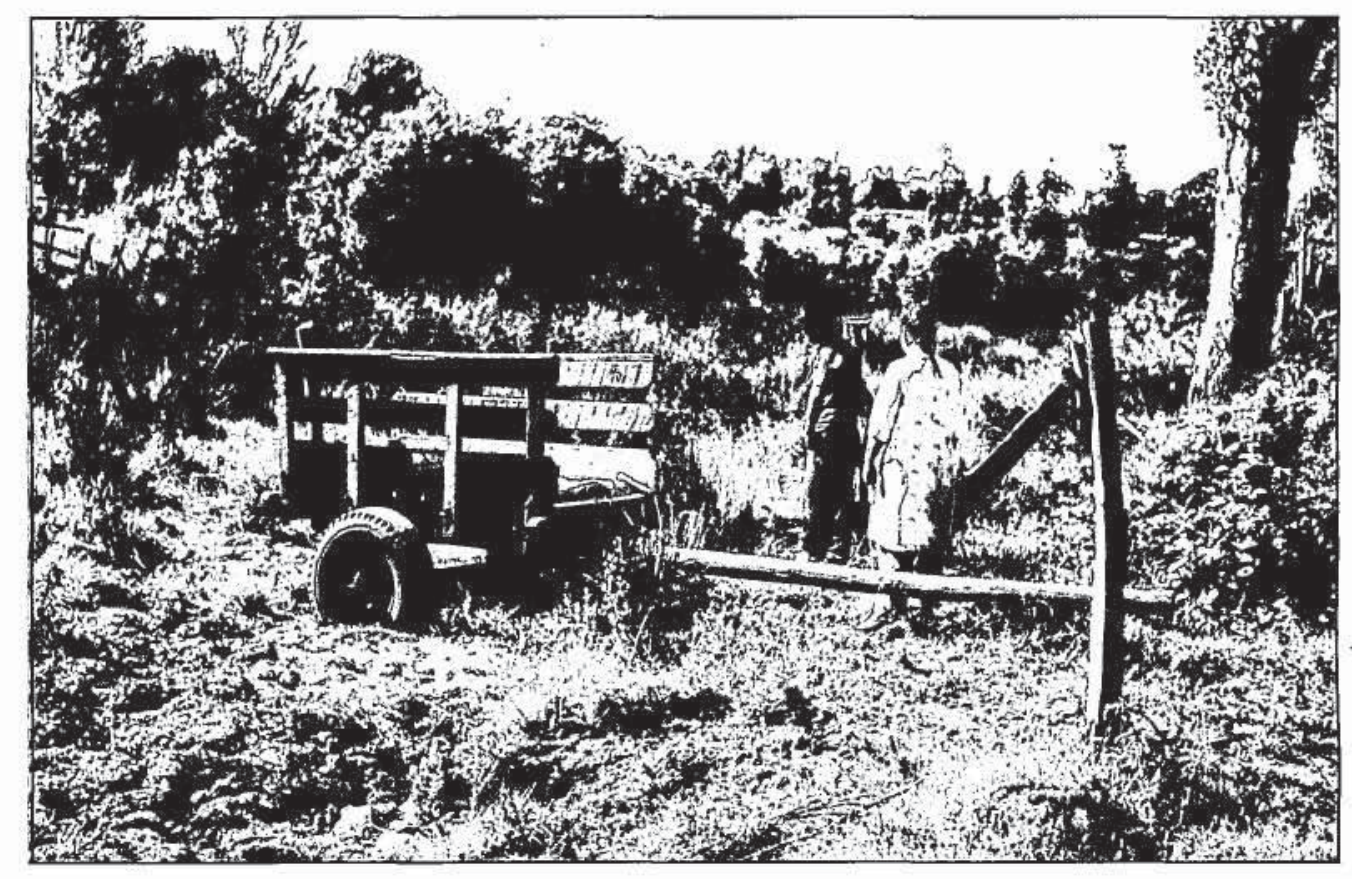

interacción, en términos de actos de comunicación y comportamientos recíprocos entre ambos.

Las posibilidades de la antropología en términos de análisis y comprensión reflexiva de los hechos y situaciones que dan forma y contenido a los comportamientos de la gente, conlleva en esta oportunidad a extrapolar los resultados de esta investigación a la situación de la sociedad y cultura que interactua con la estudiada. Se trata de poder representar una inquietud antropológica más general, dirigida a trasladar las preguntas hechas a otra cultura hacia la nuestra. ¿Cómo damos sentido, los llamados «occidentales», a la prevención de enfermedades?, ¿De qué forma enfrentamos en nuestra sociedad las situaciones de riesgo? En definitiva, ¿cómo asumimos las situaciones de prevención y riesgo en nuestra propia estructura ideacional?

Esta interrogante final, persigue la necesidad de expresar el vuelco de la mirada antropológica a nuestra sociedad, y a trata de descubrir y comprender cuáles son nuestros propios mecanismos de prevención y de atribución de la culpa.

La etnografía, como modalidad antropológica di- 
rigida hacia la búsqueda del diálogo intercultural, se constituye finalmente en los fundamentos de un proceso dialéctico que estimula el surgimiento de este tipo de interrogantes. Lo que C. Geertz llama el «estar aquí», pero agregando el sentido del transporte de las preguntas y cuestionamientos hechos a la otra cultura a la propia del etnógrafo.

Desde la perspectiva de $\mathrm{R}$. Ulin, se trataría también de un tema de racionalidades, reconocidas por el antropólogo dispuesto a asumir la búsqueda de simetrías a través del diálogo.

«Es pues por medio del encuentro reflexivo con otras culturas como la racionalidad llega a entablar un diálogo con la práctica de la antropología».(10)

BIBLIOGRAFÍA

CITARELLA,L., A.M. OYARCE, A. VIDAL, J.JELVES, AM. CONEJEROS, B. ESPINOZA. (1995). Medicinas y culturas en la araucanía. Ed. Sudamericana, Chile.

DOUGLAS, M. (1991). Pureza y peligro. Un análisis de los conceptos de contaminación y tabú. Ed. Siglo Veintiuno. España.

GEERTZ, C. (1988). La Interpretación de las culturas. Ed. Gedisa. España.

GEERTZ, C. (1989).El antropólogo como autor. Ed. Paidós. Argentina.

ULIN, R. (1990). Antropología y teoría social. Ed. Siglo Veintiuno. México.

\section{Historia}

\section{Institucional del \\ Centro de Estudios Socioculturales}

El proyecto que dio origen al CES propuso el cumplimiento de sus objetivos concibiendo la gestación de una instancia académica en proceso de permanente vitalización institucional. En el ámbito universitario ello significaría constituirse en un recurso académico virtual a toda iniciativa que considerara el componente sociocultural en su planificación, particularmente de aquellas que, en su perspectiva aplicada, hubiesen de llevarse a cabo en sectores rurales o marginales del área urbana. En el plano del trabajo especializado, la cobertura estaría dada por el surgimiento libre del proyecto a llevar a cabo desde las disciplinas involucradas y, en situaciones preferenciales, cuando la demanda emergiera de los propios contextos sociales a los cuales se pensara servir. El propósito de trasfondo no podía ser otro que generar y acumular conocimiento sociocultural y a través de ello facilitar la revisión y profundización teórica, como corresponde a todo quehacer académico (Decreto de Rectoría 30/97, Títulos I y II).

Para el cumplimiento de esta senda, la conformación del Consejo Directivo fue crucial (16 de mayo). En esta instancia estaría representada la Universidad, sus Facultades, Institutos y Escuelas, de modo de nutrir ampliamente la marcha institucional del CES, sus iniciativas específicas y la proyección de este quehacer en el espacio universitario, extra universitario, regional y externo. En sus casi dos años de evolución, el CES ha contado con un Consejo Directivo activo, preocupado 